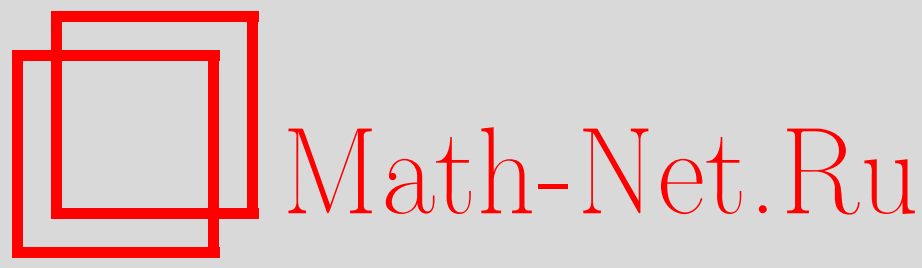

Н. М. Хатамов, Неединственность меры Гиббса для шаровой модели Изинга, ТМФ, 2014, том 180, номер 3, 318328

DOI: https://doi.org/10.4213/tmf8685

Использование Общероссийского математического портала Math-Net.Ru подразумевает, что вы прочитали и согласны с пользовательским соглашением http://www . mathnet.ru/rus/agreement

Параметры загрузки:

IP : 54.237 .59 .107

26 апреля 2023 г., $15: 25: 36$

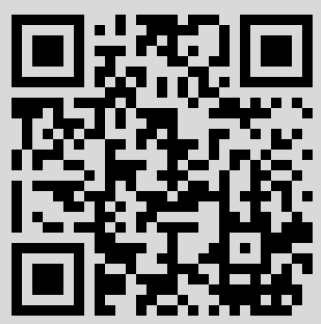




\title{
ФИЗИКА
}

Том 180, № 3

сентябрь, 2014

\section{НЕЕДИНСТВЕННОСТЬ МЕРЫ ГИББСА ДЛЯ ШАРОВОЙ МОДЕЛИ ИЗИНГА}

\begin{abstract}
Изучается новая модель - так называемая шаровая модель Изинга на дереве Кэли порядка $k \geqslant 2$. Показано, что существует критическая активность $\lambda_{\mathrm{cr}}=$ $\sqrt[4]{0.064}$ такая, что при $\lambda \geqslant \lambda_{\text {сr }}$ существует не менее одной трансляционно-инвариантной меры Гиббса, при $0<\lambda<\lambda_{\text {сr }}$ существуют не менее трех трансляционно-инвариантных мер Гиббса, а при некоторых $\lambda$ можно сказать о существовании пяти трансляционно-инвариантных мер Гиббса и континуума мер Гиббса, не являющихся трансляционно-инвариантными. Для любого нормального делителя $\widehat{G}$ индекса 2 группового представления на дереве Кэли изучены $\widehat{G}$-периодические меры Гиббса. Доказано, что существует несчетное множество $\widehat{G}$-периодических (не являющихся трансляционно-инвариантными и "шахматно"-периодическими) мер Гиббса.
\end{abstract}

Ключевые слова: дерево Кэли, конфигурация, шаровая модель Изинга, мера Гиббса.

DOI: $10.4213 / \operatorname{tmf} 8685$

\section{1. ВВЕДЕНИЕ}

Дерево Кэли $\Gamma^{k}=(V, L)$ порядка $k \geqslant 1$ - бесконечное дерево, т. е. граф без циклов, из каждой вершины которого выходит ровно $k+1$ ребер, где $V$ - множество вершин $\Gamma^{k}, L$ - его множество ребер. Пусть $i$ - функция инцидентности, сопоставляющая каждому ребру $l \in L$ его концевые точки $x, y \in V$. Если $i(l)=\{x, y\}$, то вершины $x$ и $y$ называются ближайшими соседями, и вводится обозначение $\langle x, y\rangle$. Расстояние $d(x, y), x, y \in V$, на дереве Кэли определяется формулой

$d(x, y)=\min \left\{d \mid \exists x=x_{0}, x_{1}, \ldots, x_{d-1}, x_{d}=y \in V\right.$ такое, что $\left.\left\langle x_{0}, x_{1}\right\rangle, \ldots,\left\langle x_{d-1}, x_{d}\right\rangle\right\}$.

Известно, что дерево Кэли представляется как группа $G_{k}$, являющаяся свободным произведением $k+1$ циклических групп второго порядка с образующими $a_{1}, a_{2}, \ldots, a_{k+1}[1]$.

Рассмотрим модель, где спин принимает значения из множества $\Phi=\{-1,1\}$. Тогда конфигураиия $\sigma$ на $V$ определяется как функция $x \in V \rightarrow \sigma(x) \in \Phi$; множество всех конфигураций совпадает с $\Omega=\Phi^{V}$. Пусть $A \subset V$. Обозначим через $\Omega_{A}$ пространство конфигураций, определенных на множестве $A$.

*Наманганский государственный университет, Наманган, Узбекистан.

E-mail: nxatamov@mail.ru 
Гамильтониан шаровой модели Изинга определяется следующим образом:

$$
H(\sigma)=-J \sum_{\substack{\left\langle x_{0}, x_{i}\right\rangle, i=\overline{1, k+1}}} \sigma\left(x_{0}\right) \sigma\left(x_{1}\right) \sigma\left(x_{2}\right) \ldots \sigma\left(x_{k+1}\right),
$$

где $J>0$.

Обозначим через $M$ множество всех шаров $b(x)=\{y \in V \mid d(x, y) \leqslant 1\}$ с радиусом 1. Будем называть сужение конфигурации $\sigma$ на шаре $b \in M$ ограниченной конфигурацией $\sigma_{b}$. Тогда определим функцию $U\left(\sigma_{b}\right): \Omega_{b} \rightarrow \Phi$ следующим образом:

$$
U\left(\sigma_{b}\right)=\sigma\left(x_{0}\right) \sigma\left(x_{1}\right) \sigma\left(x_{2}\right) \ldots \sigma\left(x_{k+1}\right),
$$

где $\Omega_{b}$ - пространство конфигураций $\sigma_{b}$, а $x_{0}, x_{i} \in V$, такие, что $\left\langle x_{0}, x_{i}\right\rangle, i=\overline{1, k+1}$.

Таким образом, гамильтониан (1) мы можем записать в следующем виде:

$$
H(\sigma)=-J \sum_{b \in M} U\left(\sigma_{b}\right)
$$

где $J \in \mathbb{R}$.

Наиболее сложная задача в случае данного гамильтониана - это описание всех отвечающих ему мер Гиббса. Определение меры Гиббса и других понятий, связанных с теорией гиббсовских мер, можно найти, например, в [2]-[4].

\section{2. СИСТЕМА ФУНКЦИОНАЛЬНЫХ УРАВНЕНИЙ}

Пусть $x^{0} \in V$ - фиксированная точка. Будем писать $x<y$, если путь от $x^{0}$ до $y$ проходит через $x$, и тогда будем говорить, что $x$ и $y$ являются соседями. Вершина $y$ называется потомком $x$, если $y>x$ и $x, y$ являются соседями. Точка $y$ называется прямым потомком точки $x$, если $y>x$ и $x, y$ являются ближайшими соседями, т. е. $d(x, y)=1$.

Введем обозначения

$$
W_{n}=\left\{x \in V \mid d\left(x^{0}, x\right)=n\right\}, \quad V_{n}=\left\{x \in V \mid d\left(x^{0}, x\right) \leqslant n\right\} .
$$

Для $x \in G_{k}$ введем обозначение $x_{\downarrow}=\left\{y \in G_{k} \mid\langle x, y\rangle\right\} \backslash S(x)$, где $S(x)$ - множество прямых потомков точки $x \in V$.

Рассмотрим случай $k=2$. Пусть

$$
b(x)=\left\{x, x a_{1}, x a_{2}, x a_{3}\right\}, \quad \sigma_{b(x)}=\left\{\sigma(x), \sigma\left(x a_{1}\right), \sigma\left(x a_{2}\right), \sigma\left(x a_{3}\right)\right\} .
$$

Вероятностное распределение $\mu^{(n)}$ на $\Omega_{V_{n}}$ имеет вид

$$
\mu^{(n)}\left(\sigma_{n}\right)=Z_{n}^{-1} \exp \left\{-\beta H\left(\sigma_{n}\right)+\sum_{x \in W_{n}} h_{b(x), \sigma_{b}(x)}^{\sigma_{b}\left(x_{\downarrow}\right)}\right\},
$$

где $\sigma_{n} \in \Omega_{V_{n}}$,

$$
Z_{n}=\sum_{\bar{\sigma}_{n} \in \Omega_{V_{n}}} \exp \left\{-\beta H\left(\bar{\sigma}_{n}\right)+\sum_{x \in W_{n}} h_{b(x), \bar{\sigma}_{b}(x)}^{\bar{\sigma}_{b}\left(x_{\downarrow}\right)}\right\}
$$

и $h_{b, \bar{\sigma}}^{\bar{\sigma}} \in \mathbb{R}$. 
Говорят, что вероятностное распределение $\mu^{(n)}$ для всех $n \geqslant 1$ согласованно, если

$$
\sum_{\sigma^{(n)}} \mu^{(n)}\left(\sigma_{n-1}, \sigma^{(n)}\right)=\mu^{(n-1)}\left(\sigma_{n-1}\right)
$$

для всех $n \geqslant 1$ и $\sigma_{n-1} \in \Omega_{V_{n-1}}$. В этом случае существует единственная мера $\mu$ на $\Omega_{V}$ такая, что

$$
\mu\left(\left\{\left.\sigma\right|_{V_{n}}=\sigma_{n}\right\}\right)=\mu^{(n)}\left(\sigma_{n}\right)
$$

для всех $n \geqslant 1$ и $\sigma_{n} \in \Omega_{V_{n}}$.

Рассмотрим конфигурации

$$
\begin{aligned}
\sigma_{0} & =\{+,+,+,+\}, \quad \sigma_{1}=\{+,-,+,+\}, \\
\sigma_{2} & =\{+,+,-,-\}, \quad \sigma_{3}=\{+,-,-,-\}, \\
-\sigma_{0} & =\{-,-,-,-\}, \quad-\sigma_{1}=\{-,+,-,-\}, \\
-\sigma_{2} & =\{-,-,+,+\}, \quad-\sigma_{3}=\{-,+,+,+\}
\end{aligned}
$$

на единичном шаре. Обозначим

$$
\begin{array}{rlrl}
h_{b, \sigma_{0}}^{\sigma_{0}} & =h_{b, 0}, \quad h_{b, \sigma_{1}}^{\sigma_{0}}=h_{b, 1}, \quad h_{b, \sigma_{2}}^{\sigma_{0}}=h_{b, 2}, \quad h_{b,-\sigma_{1}}^{\sigma_{1}}=h_{b, 3}, \\
h_{b,-\sigma_{2}}^{\sigma_{1}} & =h_{b, 4}, \quad h_{b,-\sigma_{3}}^{\sigma_{1}}=h_{b, 5}, \quad h_{b, \sigma_{1}}^{-\sigma_{1}}=h_{b, 6}, & h_{b, \sigma_{2}}^{-\sigma_{1}}=h_{b, 7}, \\
h_{b, \sigma_{3}}^{-\sigma_{1}} & =h_{b, 8}, \quad h_{b,-\sigma_{0}}^{-\sigma_{0}}=h_{b, 9}, \quad h_{b,-\sigma_{1}}^{-\sigma_{0}}=h_{b, 10}, \quad h_{b,-\sigma_{2}}^{-\sigma_{0}}=h_{b, 11} .
\end{array}
$$

Пусть $k=2$. Для $a \in M$ через $b, c$ обозначим прямых потомков шара (см. [2]).

Следующая теорема доказывается аналогично теореме 1 из работы [5]. Эта теорема дает необходимые и достаточные условия на $h_{b, i}$, при которых выполняется соотношение (5).

Теорема 1. Пусть $k=2$. Вероятностное распределение $\mu^{(n)}\left(\sigma_{n}\right), n=1,2, \ldots$, в (4) согласованно тогда и только тогда, когда для любого а $\in$ М имеют место следующие равенства:

$$
\begin{aligned}
& y_{a, 0}=y_{a, 6}=\frac{y_{b, 0}+\lambda^{2} y_{b, 1}+y_{b, 2}}{\lambda^{2} y_{b, 0}+y_{b, 1}+\lambda^{2} y_{b, 2}} \frac{y_{c, 0}+\lambda^{2} y_{c, 1}+y_{c, 2}}{\lambda^{2} y_{c, 0}+y_{c, 1}+\lambda^{2} y_{c, 2}}, \\
& y_{a, 1}=y_{a, 7}=\frac{y_{b, 0}+\lambda^{2} y_{b, 1}+y_{b, 2}}{\lambda^{2} y_{b, 0}+y_{b, 1}+\lambda^{2} y_{b, 2}} \frac{\lambda^{2} y_{c, 3}+y_{c, 4}+\lambda^{2}}{\lambda^{2} y_{c, 0}+y_{c, 1}+\lambda^{2} y_{c, 2}}, \\
& y_{a, 2}=y_{a, 8}=\frac{\lambda^{2} y_{b, 3}+y_{b, 4}+\lambda^{2}}{\lambda^{2} y_{b, 0}+y_{b, 1}+\lambda^{2} y_{b, 2}} \frac{\lambda^{2} y_{c, 3}+y_{c, 4}+\lambda^{2}}{\lambda^{2} y_{c, 0}+y_{c, 1}+\lambda^{2} y_{c, 2}}, \\
& y_{a, 3}=y_{a, 9}=\frac{y_{b, 3}+\lambda^{2} y_{b, 4}+1}{\lambda^{2} y_{b, 0}+y_{b, 1}+\lambda^{2} y_{b, 2}} \frac{y_{c, 3}+\lambda^{2} y_{c, 4}+1}{\lambda^{2} y_{c, 0}+y_{c, 1}+\lambda^{2} y_{c, 2}}, \\
& y_{a, 4}=y_{a, 10}=\frac{y_{c, 3}+\lambda^{2} y_{c, 4}+1}{\lambda^{2} y_{c, 0}+y_{c, 1}+\lambda^{2} y_{c, 2}}, \quad y_{a, 5}=1,
\end{aligned}
$$

где $\lambda=e^{J \beta}, \beta=1 / T, y_{a, i}=e^{h_{a, i}-h_{a, 11}}, i=\overline{0,10}$. 


\section{3. ТРАНСЛЯЦИОННО-ИНВАРИАНТНЫЕ МЕРЫ ГИББСА}

В этом разделе изучаются трансляционно-инвариантные меры Гиббса для модели (3) на дереве Кэли. Заметим, что трансляционно-инвариантные меры Гиббса соответствуют решениям (7) с $y_{a, i}=y_{i}$ при всех $a \in M$ и $i=0,1,2,3,4$.

В (7) положим $y_{a, i}=y_{i} \in \mathbb{R}_{+}$при всех $a \in M$ и получим

$$
\begin{aligned}
& y_{0}=\left(\frac{y_{0}+\lambda^{2} y_{1}+y_{2}}{\lambda^{2} y_{0}+y_{1}+\lambda^{2} y_{2}}\right)^{2}, \\
& y_{1}=\left(\frac{y_{0}+\lambda^{2} y_{1}+y_{2}}{\lambda^{2} y_{0}+y_{1}+\lambda^{2} y_{2}}\right)\left(\frac{\lambda^{2} y_{3}+y_{4}+\lambda^{2}}{\lambda^{2} y_{0}+y_{1}+\lambda^{2} y_{2}}\right), \\
& y_{2}=\left(\frac{\lambda^{2} y_{3}+y_{4}+\lambda^{2}}{\lambda^{2} y_{0}+y_{1}+\lambda^{2} y_{2}}\right)^{2}, \\
& y_{3}=\left(\frac{y_{3}+\lambda^{2} y_{4}+1}{\lambda^{2} y_{0}+y_{1}+\lambda^{2} y_{2}}\right)^{2}, \\
& y_{4}=\frac{y_{3}+\lambda^{2} y_{4}+1}{\lambda^{2} y_{0}+y_{1}+\lambda^{2} y_{2}},
\end{aligned}
$$

где $\lambda>0$.

ЛЕмма 1. Систему уравнений (8) можно привести к виду

$$
\begin{aligned}
f(y, \lambda)=( & \left.\lambda^{2}\left(y^{2}+\lambda^{2} y+1\right)^{2}-\left(\lambda^{4}-1\right) y^{2}\left(\lambda^{2} y^{2}+y+\lambda^{2}\right)\right)^{2}\left(y^{2}+\lambda^{2} y+1\right)^{3} \times \\
& \times\left[\lambda^{2}\left(y^{2}+\lambda^{2} y+1\right)^{6}+y\left(\lambda^{2}\left(y^{2}+\lambda^{2} y+1\right)^{2}-\right.\right. \\
& \left.-\left(\lambda^{4}-1\right) y^{2}\left(\lambda^{2} y^{2}+y+\lambda^{2}\right)\right)\left(\lambda^{2} y^{2}+y+\lambda^{2}\right)\left(y^{2}+\lambda^{2} y+1\right)^{3}+ \\
& +y^{2} \lambda^{2}\left(\lambda^{2} y^{2}+y+\lambda^{2}\right)^{2}\left(\lambda^{2}\left(y^{2}+\lambda^{2} y+1\right)^{2}-\right. \\
& \left.\left.-\left(\lambda^{4}-1\right) y^{2}\left(\lambda^{2} y^{2}+y+\lambda^{2}\right)\right)^{2}\right]^{-1}-y=0,
\end{aligned}
$$

где $y=y_{4}$.

ДокАЗАТЕЛЬСТво. Заметим, что если $y_{4}=y$, то $y_{3}=y^{2}$. Из пятого уравнения системы (8) получаем

$$
\lambda^{2} y_{0}+y_{1}+\lambda^{2} y_{2}=\frac{y^{2}+\lambda^{2} y+1}{y} .
$$

Тогда

$$
y_{0}+y_{2}=\frac{y^{2}+\lambda^{2} y+1}{\lambda^{2} y}-\frac{y_{1}}{\lambda^{2}} .
$$

Используя равенства (10) и (11), из второго уравнения системы (8) имеем

$$
y_{1}=\frac{y\left(\lambda^{2} y^{2}+y+\lambda^{2}\right)\left(y^{2}+\lambda^{2} y+1\right)^{2}}{\lambda^{2}\left(y^{2}+\lambda^{2} y+1\right)^{2}-\left(\lambda^{4}-1\right) y^{2}\left(\lambda^{2} y^{2}+y+\lambda^{2}\right)} .
$$

Тогда получим

$$
y_{0}+\lambda^{2} y_{1}+y_{2}=\frac{\left(y^{2}+\lambda^{2} y+1\right)^{3}}{y\left(\lambda^{2}\left(y^{2}+\lambda^{2} y+1\right)^{2}-\left(\lambda^{4}-1\right) y^{2}\left(\lambda^{2} y^{2}+y+\lambda^{2}\right)\right)} .
$$

2 Теоретическая и математическая физика, т. 180, № 3, 2014 г. 


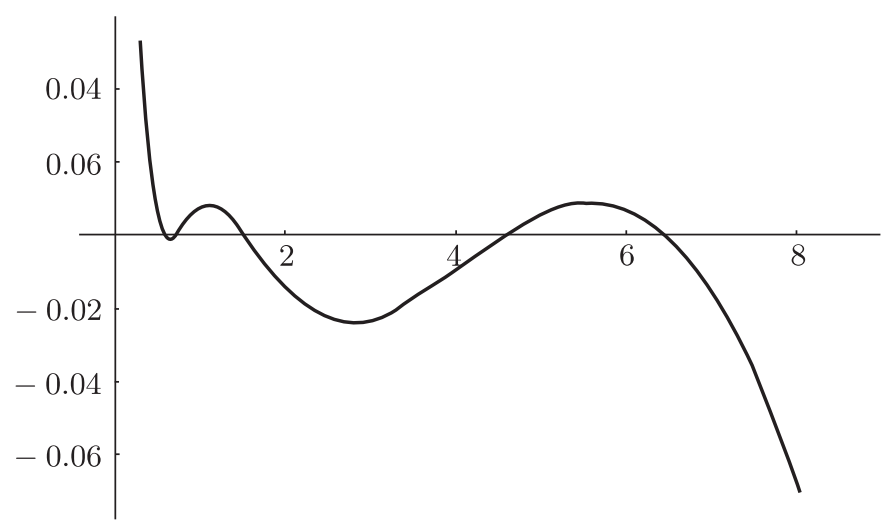

Рис. 1. График функции $f(y, \lambda)$ при $\lambda=0.53$.

Умножая на $\lambda^{2}$ первое и третье уравнения системы (8), сложим первое, второе и третье уравнения из той же системы. Используя полученные выше равенства, получим (9). Лемма доказана.

Теорема 2. Для модели (3) существует $\lambda_{\mathrm{cr}}=\sqrt[4]{0.064}$ maкое, что при $\lambda \geqslant \lambda_{\mathrm{cr}}$ существует не менее одной трансляционно-инвариантной меры Гиббса, при $0<$ $\lambda<\lambda_{\text {сг }}$ существуют не менее трех транслящионно-инвариантных мер Гиббса.

ДоказАтельство. Рассмотрим свойства функции $f(y, \lambda)$ :

1) $f(0, \lambda)=\lambda^{2}>0$

2) $f(+\infty, \lambda)=-\infty<0$.

Тогда ясно, что при любых $\lambda>0$ существует по крайней мере одно решение уравнения $f(y, \lambda)=0$.

Можно доказать, что $\left(\lambda^{-2 / 3}, \lambda\right)$ является решением уравнения $f(y, \lambda)=0$ (см. ниже доказательство теоремы 4). В этой точке рассмотрим значение функции $f_{y}^{\prime}(y, \lambda)$, получим

$$
f_{y}^{\prime}\left(\lambda^{-2 / 3}, \lambda\right)=-\frac{3 \lambda^{8 / 3}\left(5 \lambda^{4 / 3}-2\right)}{\left(1-\lambda^{1 / 3}+\lambda^{2 / 3}\right)^{2}\left(1+\lambda^{1 / 3}+\lambda^{2 / 3}\right)^{2}\left(1-\lambda^{2 / 3}+\lambda^{4 / 3}\right)^{2}}
$$

Если $f_{y}^{\prime}\left(\lambda^{-2 / 3}, \lambda\right)>0$, т. е. $0<\lambda<\sqrt[4]{0.064}$, то уравнение $f(y, \lambda)=0$ имеет не менее трех решений. Теорема доказана.

При некоторых $\lambda \in(0,+\infty)$ могут существовать другие трансляционно-инвариантные меры, кроме описанных в теореме 2. Например, рис. 1 демонстрирует существование не менее пяти трансляционно-инвариантных мер Гиббса при $\lambda=0.53$.

Действительно, численный анализ показывает, что, например, при $\lambda=0.53$ решениями уравнения $f_{y}^{\prime}(y, 0.53)=0$ являются следующие значения: $y_{1}=0.642531$, $y_{2}=1.10499, y_{3}=2.77189, y_{4}=5.57841$. Точки $\left(y_{i}, 0.53\right), i=\overline{1,4}$, являются критическими точками функции $f(y, 0.53)$. 
Вычисляя значения функции в этих точках, получим

$$
\begin{aligned}
& f\left(y_{1}, 0.53\right)=-0.008561695<0 \\
& f\left(y_{2}, 0.53\right)=0.008113591>0 \\
& f\left(y_{3}, 0.53\right)=-0.23894615<0 \\
& f\left(y_{4}, 0.53\right)=0.008776144>0 .
\end{aligned}
$$

Тогда можно заключить, что при $\lambda=0.53$ уравнение $f(y, \lambda)=0$ имеет не менее пяти решений. Анализ показывает, что интервал таких $\lambda$ есть $\left(\lambda_{\mathrm{cr}}, 0.54\right)$.

Применяя методы работ [6], [7], легко доказывается следующая

Теорема 3. При $0<\lambda<\lambda_{\text {сг }}$ существует континуум мер Гиббса, не являющихся трансляционно-инвариантными.

Предположим, что

$$
y_{a, 0}=y_{a, 3}, \quad y_{a, 1}=y_{a, 4}, \quad y_{a, 2}=1 \quad \forall a \in M
$$

Тогда из (7) следует

$$
\begin{aligned}
y_{a, 0} & =\frac{y_{b, 0}+\lambda^{2} y_{b, 1}+1}{\lambda^{2} y_{b, 0}+y_{b, 1}+\lambda^{2}} \frac{y_{c, 0}+\lambda^{2} y_{c, 1}+1}{\lambda^{2} y_{c, 0}+y_{c, 1}+\lambda^{2}}, \\
y_{a, 1} & =\frac{y_{b, 0}+\lambda^{2} y_{b, 1}+1}{\lambda^{2} y_{b, 0}+y_{b, 1}+\lambda^{2}} .
\end{aligned}
$$

Имеем следующую лемму.

Лемма 2. А. Пусть $\lambda>1$. Если $y_{a}=\left(y_{a, 0}, y_{a, 1}\right), a \in M$, является решением системы (15), то $y_{i}^{-} \leqslant y_{a, i} \leqslant y_{i}^{+}$для любого $i=0,1, a \in M$, где $\left(y_{0}^{-}, y_{0}^{+}, y_{1}^{-}, y_{1}^{+}\right)-$ решения системь

$$
\begin{aligned}
& y_{0}^{-}=\left(\frac{y_{0}^{-}+\lambda^{2} y_{1}^{+}+1}{\lambda^{2} y_{0}^{-}+y_{1}^{+}+\lambda^{2}}\right)^{2}, \\
& y_{0}^{+}=\left(\frac{y_{0}^{+}+\lambda^{2} y_{1}^{-}+1}{\lambda^{2} y_{0}^{+}+y_{1}^{-}+\lambda^{2}}\right)^{2}, \\
& y_{1}^{-}=\frac{y_{0}^{-}+\lambda^{2} y_{1}^{+}+1}{\lambda^{2} y_{0}^{-}+y_{1}^{+}+\lambda^{2}}, \\
& y_{1}^{+}=\frac{y_{0}^{+}+\lambda^{2} y_{1}^{-}+1}{\lambda^{2} y_{0}^{+}+y_{1}^{-}+\lambda^{2}} .
\end{aligned}
$$

Б. Пусть $\lambda<1$. Если $y_{a}=\left(y_{a, 0}, y_{a, 1}\right), a \in M$, является решением системь (15), то $y_{i}^{-} \leqslant y_{a, i} \leqslant y_{i}^{+}$для любого $i=0,1, a \in M$, где $\left(y_{0}^{-}, y_{0}^{+}, y_{1}^{-}, y_{1}^{+}\right)$- решения системь

$$
\begin{aligned}
& y_{0}^{-}=\left(\frac{y_{0}^{+}+\lambda^{2} y_{1}^{-}+1}{\lambda^{2} y_{0}^{+}+y_{1}^{-}+\lambda^{2}}\right)^{2}, \\
& y_{0}^{+}=\left(\frac{y_{0}^{-}+\lambda^{2} y_{1}^{+}+1}{\lambda^{2} y_{0}^{-}+y_{1}^{+}+\lambda^{2}}\right)^{2},
\end{aligned}
$$




$$
\begin{aligned}
& y_{1}^{-}=\frac{y_{0}^{+}+\lambda^{2} y_{1}^{-}+1}{\lambda^{2} y_{0}^{+}+y_{1}^{-}+\lambda^{2}}, \\
& y_{1}^{+}=\frac{y_{0}^{-}+\lambda^{2} y_{1}^{+}+1}{\lambda^{2} y_{0}^{-}+y_{1}^{+}+\lambda^{2}} .
\end{aligned}
$$

ДоказАтельство. А. Пусть $\lambda>1$. Очевидно, что $0<y_{a, 0}<\lambda^{4}, 0<y_{a, 1}<\lambda^{2}$ для любого $a \in M$.

Рассмотрим функцию

$$
f(u, v)=\frac{u+\lambda^{2} v+1}{\lambda^{2} u+v+\lambda^{2}}
$$

где $0<u<\lambda^{4}, 0<v<\lambda^{2}$. Нетрудно видеть, что $\left(2 \lambda^{2}+1\right) / 2 \lambda^{2}<f(u, v)<\lambda^{2}$. Таким образом, для $y_{a, i}$ мы получаем

$$
\left(\frac{2 \lambda^{2}+1}{2 \lambda^{2}}\right)^{2}<y_{a, 0}<\lambda^{2}, \quad \frac{2 \lambda^{2}+1}{2 \lambda^{2}}<y_{a, 1}<\lambda^{2} .
$$

Теперь рассмотрим функцию $f(u, v)$ с условиями

$$
\left(\frac{2 \lambda^{2}+1}{2 \lambda^{2}}\right)^{2}<u<\lambda^{4}, \quad \frac{2 \lambda^{2}+1}{2 \lambda^{2}}<v<\lambda^{2} .
$$

Итерируя эту процедуру, мы получим $y_{i, n}^{-} \leqslant y_{a, i} \leqslant y_{i, n}^{+}, i=0,1$, где $y_{i, n}^{ \pm}, i=0,1$, $n=1,2, \ldots$, удовлетворяют равенствам

$$
\begin{aligned}
& y_{0, n+1}^{-}=\left(\frac{y_{0, n}^{-}+\lambda^{2} y_{1, n}^{+}+1}{\lambda^{2} y_{0, n}^{-}+y_{1, n}^{+}+\lambda^{2}}\right)^{2}, \\
& y_{0, n+1}^{+}=\left(\frac{y_{0, n}^{+}+\lambda^{2} y_{1, n}^{-}+1}{\lambda^{2} y_{0, n}^{+}+y_{1, n}^{-}+\lambda^{2}}\right)^{2}, \\
& y_{1, n+1}^{-}=\frac{y_{0, n}^{-}+\lambda^{2} y_{1, n}^{+}+1}{\lambda^{2} y_{0, n}^{-}+y_{1, n}^{+}+\lambda^{2}}, \\
& y_{1, n+1}^{+}=\frac{y_{0, n}^{+}+\lambda^{2} y_{1, n}^{-}+1}{\lambda^{2} y_{0, n}^{+}+y_{1, n}^{-}+\lambda^{2}},
\end{aligned}
$$

где $y_{0,1}^{-}=0, y_{0,1}^{+}=\lambda^{4}, y_{1,1}^{-}=0, y_{1,1}^{+}=\lambda^{2}$. Видно, что $y_{i, n}^{-} \quad\left(y_{i, n}^{+}\right), i=0,1$, являются возрастающими (убывающими) и ограниченными последовательностями. Поэтому существуют $\lim _{x \rightarrow \infty} y_{i, n}^{ \pm}=y_{i}^{ \pm}, i=0,1$.

Б. Пусть $\lambda<1$. Очевидно, что $0<y_{a, 0}<1 / \lambda^{4}, 0<y_{a, 1}<1 / \lambda^{2}$ для любого $a \in M$. Рассмотрим функцию

$$
f(u, v)=\frac{u+\lambda^{2} v+1}{\lambda^{2} u+v+\lambda^{2}}
$$

с условиями $0<u<1 / \lambda^{4}, 0<v<1 / \lambda^{2}$. Видно, что $2 /\left(\lambda^{2}+1 / \lambda^{2}\right)<f(u, v)<1 / \lambda^{2}$. Следовательно, для $y_{a, i}$ мы получаем

$$
\left(\frac{2}{\lambda^{2}+1 / \lambda^{2}}\right)^{2}<y_{a, 0}<\frac{1}{\lambda^{4}}, \quad \frac{2}{\lambda^{2}+1 / \lambda^{2}}<y_{a, 1}<\frac{1}{\lambda^{2}} .
$$


Теперь рассмотрим функцию $f(u, v)$ с условиями

$$
\left(\frac{2}{\lambda^{2}+1 / \lambda^{2}}\right)^{2}<u<\frac{1}{\lambda^{4}}, \quad \frac{2}{\lambda^{2}+1 / \lambda^{2}}<v<\frac{1}{\lambda^{2}} .
$$

Итерируя эту процедуру, мы можем получить $y_{i, n}^{-} \leqslant y_{a, i} \leqslant y_{i, n}^{+}, i=0,1$, где $y_{i, n}^{ \pm}$, $i=0,1, n=1,2, \ldots$, удовлетворяют равенствам

$$
\begin{aligned}
& y_{0, n+1}^{-}=\left(\frac{y_{0, n}^{+}+\lambda^{2} y_{1, n}^{-}+1}{\lambda^{2} y_{0, n}^{+}+y_{1, n}^{-}+\lambda^{2}}\right)^{2}, \\
& y_{0, n+1}^{+}=\left(\frac{y_{0, n}^{-}+\lambda^{2} y_{1, n}^{+}+1}{\lambda^{2} y_{0, n}^{-}+y_{1, n}^{+}+\lambda^{2}}\right)^{2}, \\
& y_{1, n+1}^{-}=\frac{y_{0, n}^{+}+\lambda^{2} y_{1, n}^{-}+1}{\lambda^{2} y_{0, n}^{+}+y_{1, n}^{-}+\lambda^{2}}, \\
& y_{1, n+1}^{+}=\frac{y_{0, n}^{-}+\lambda^{2} y_{1, n}^{+}+1}{\lambda^{2} y_{0, n}^{-}+y_{1, n}^{+}+\lambda^{2}},
\end{aligned}
$$

где $y_{0,1}^{+}=0, y_{0,1}^{-}=1 / \lambda^{4}, y_{1,1}^{+}=0, y_{1,1}^{-}=1 / \lambda^{2}$. Отсюда видно, что $y_{i, n}^{-}\left(y_{i, n}^{+}\right), i=0,1$, являются возрастающими (убывающими) и ограниченными последовательностями. Поэтому существуют $\lim _{n \rightarrow \infty} y_{i, n}^{ \pm}, i=0,1$. Лемма доказана.

Лемма 3. Если $y=\left(y_{0}^{-}, y_{0}^{+}, y_{1}^{-}, y_{1}^{+}\right)$- решение системы (16), то $y_{0}^{-}=y_{0}^{+}$тогда и толъко тогда, когда $y_{1}^{-}=y_{1}^{+}$.

ДоКАЗАТЕЛЬСтво. Из системы (16) мы имеем

$$
\begin{array}{r}
\left(y_{0}^{-}-y_{0}^{+}\right)\left(1+A B y_{1}^{+}\right)+A B\left(1+y_{0}^{-}\right)\left(y_{1}^{-}-y_{1}^{+}\right)=0 \\
\left(y_{0}^{-}-y_{0}^{+}\right) y_{1}^{+} B+\left(B+1+B y_{0}^{-}\right)\left(y_{1}^{-}-y_{1}^{+}\right)=0
\end{array}
$$

где

$$
\begin{aligned}
& A=\frac{y_{0}^{-}+\lambda^{2} y_{1}^{+}+1}{\lambda^{2} y_{0}^{-}+y_{1}^{+}+\lambda^{2}}+\frac{y_{0}^{+}+\lambda^{2} y_{1}^{-}+1}{\lambda^{2} y_{0}^{+}+y_{1}^{-}+\lambda^{2}}>0, \\
& B=\frac{\lambda^{4}-1}{\left(\lambda^{2} y_{0}^{-}+y_{1}^{+}+\lambda^{2}\right)\left(\lambda^{2} y_{0}^{+}+y_{1}^{-}+\lambda^{2}\right)}>0 .
\end{aligned}
$$

Если $y_{0}^{-}=y_{0}^{+}$, то из первого уравнения системы (17) имеем $y_{1}^{-}=y_{1}^{+}$. Если $y_{1}^{-}=y_{1}^{+}$, то из второго уравнения системы (17) имеем $y_{0}^{-}=y_{0}^{+}$. Лемма доказана.

\section{4. ПЕРИОДИЧЕСКИЕ МЕРЫ ГИББСА}

В этом разделе при условии (14) изучим периодические меры Гиббса. Запишем систему (15) в следующем виде:

$$
\begin{aligned}
h_{a, 0} & =\ln \left(\frac{e^{h_{b, 0}}+\lambda^{2} e^{h_{b, 1}}+1}{\lambda^{2} e^{h_{b, 0}}+e^{h_{b, 1}}+\lambda^{2}} \frac{e^{h_{c, 0}}+\lambda^{2} e^{h_{c, 1}}+1}{\lambda^{2} e^{h_{c, 0}}+e^{h_{c, 1}}+\lambda^{2}}\right), \\
h_{a, 1} & =\ln \frac{e^{h_{b, 0}}+\lambda^{2} e^{h_{b, 1}}+1}{\lambda^{2} e^{h_{b, 0}}+e^{h_{b, 1}}+\lambda^{2}}
\end{aligned}
$$

где $h_{a, i}=\ln y_{a, i}, i=0,1$. Исследуем периодические решения системы (18). 
ОПРЕДЕЛЕНИЕ 1 . Пусть $H_{0}-$ подгруппа группы $G_{k}$. Совокупность векторов $h=$ $\left\{h_{a}=\left(h_{a, 0}, h_{a, 1}\right) \mid a \in G_{k}\right\}$ называется $H_{0}$-периодической, если $h_{y a, i}=h_{a, i}$ для любых $a \in G_{k}$ и $y \in H_{0}, i=0,1$.

ОПРЕДЕЛЕНиЕ 2. Мера Гиббса, соответствующая $H_{0}$-периодической совокупности векторов, называется $H_{0}$-периодической.

Определим $F: h=\left(h_{0}, h_{1}\right) \rightarrow F(h) \in \mathbb{R}$ следующим образом:

$$
F(h)=\frac{e^{h_{0}}+\lambda^{2} e^{h_{1}}+1}{\lambda^{2} e^{h_{0}}+e^{h_{1}}+\lambda^{2}} .
$$

Пусть $G_{k}^{(2)}$ - подгруппа группы $G_{k}$, состоящая из слов четной длины. Очевидно, что $G_{k}^{(2)}$ является подгруппой индекса 2.

Пусть

$$
h_{a, i}=\left\{\begin{array}{ll}
h_{i}, & \text { если } c_{a} \in G_{2}^{(2)}, \\
h_{i}^{\prime}, & \text { если } c_{a} \in G_{2} \backslash G_{2}^{(2)},
\end{array} \quad i=0,1,\right.
$$

где $c_{a}$ - центр шара $a$. Тогда из (18) имеем

$$
\begin{aligned}
& h_{0}=2 \ln \frac{e^{h_{0}^{\prime}}+\lambda^{2} e^{h_{1}^{\prime}}+1}{\lambda^{2} e^{h_{0}^{\prime}}+e^{h_{1}^{\prime}}+\lambda^{2}}, \\
& h_{0}^{\prime}=2 \ln \frac{e^{h_{0}}+\lambda^{2} e^{h_{1}}+1}{\lambda^{2} e^{h_{0}}+e^{h_{1}}+\lambda^{2}}, \\
& h_{1}=\ln \frac{e^{h_{0}^{\prime}}+\lambda^{2} e^{h_{1}^{\prime}}+1}{\lambda^{2} e^{h_{0}^{\prime}}+e^{h_{1}^{\prime}}+\lambda^{2}}, \\
& h_{1}^{\prime}=\ln \frac{e^{h_{0}}+\lambda^{2} e^{h_{1}}+1}{\lambda^{2} e^{h_{0}}+e^{h_{1}}+\lambda^{2}} .
\end{aligned}
$$

Теорема 4. Для модели (3) при $\lambda>0$ и при условиях (14) существует единственная $G_{2}^{(2)}$-периодическая мера Гиббса. Более того, эта мера совпадает с транслячионно-инвариантной.

ДоказАтельство. Рассмотрим случай $h_{0}=h_{0}^{\prime}, h_{1}=h_{1}^{\prime}$. Из (19) имеем

$$
\begin{aligned}
& h_{0}=2 \ln \frac{e^{h_{0}}+\lambda^{2} e^{h_{1}}+1}{\lambda^{2} e^{h_{0}}+e^{h_{1}}+\lambda^{2}}, \\
& h_{1}=\ln \frac{e^{h_{0}}+\lambda^{2} e^{h_{1}}+1}{\lambda^{2} e^{h_{0}}+e^{h_{1}}+\lambda^{2}} .
\end{aligned}
$$

Система уравнений (20) имеет единственное решение $\left(h_{0}^{*}, h_{1}^{*}\right)$. Действительно, из $(20)$ имеем $\lambda^{2} e^{3 h_{1}}-1=0$. Пусть $e^{h_{1}}=z$, тогда $Q(z)=\lambda^{2} z^{3}-1=0$. Следовательно, уравнение $Q(z)=0$ имеет единственное положительное решение $z^{*}=1 / \sqrt[3]{\lambda^{2}}$, т. е. система (20) имеет единственное решение $\left(h_{0}^{*}, h_{1}^{*}\right)$, где $h_{0}^{*}=2 \ln \left(1 / \sqrt[3]{\lambda^{2}}\right), h_{1}^{*}=1 / \sqrt[3]{\lambda^{2}}$.

Теперь рассмотрим случай $h_{0} \neq h_{0}^{\prime}, h_{1} \neq h_{1}^{\prime}$. Из (19) имеем

$$
\begin{aligned}
& h_{1}=\ln \frac{e^{2 h_{1}^{\prime}}+\lambda^{2} e^{h_{1}^{\prime}}+1}{\lambda^{2} e^{2 h_{1}^{\prime}}+e^{h_{1}^{\prime}}+\lambda^{2}}, \\
& h_{1}^{\prime}=\ln \frac{e^{2 h_{1}}+\lambda^{2} e^{h_{1}}+1}{\lambda^{2} e^{2 h_{1}}+e^{h_{1}}+\lambda^{2}} .
\end{aligned}
$$


Из (21) получим многочлен (при $z=e^{h_{1}}$ )

$$
P(z)=\left(\lambda^{6}+2 \lambda^{2}\right) z^{5}+3 \lambda^{4} z^{4}+\left(2 \lambda^{6}+\lambda^{2}\right) z^{3}-\left(\lambda^{4}+2\right) z^{2}-3 \lambda^{2} z-\left(2 \lambda^{4}+1\right)=0 .
$$

Легко получим разложение $P(z)=Q(z) \cdot M(z)$, где $M(z)=\left(\lambda^{4}+2\right) z^{2}+3 \lambda^{2} z+2 \lambda^{4}+1$.

Так как уравнение $Q(z)=0$ имеет единственное решение и уравнение $M(z)=0$ не имеет решения, то $P(z)=0$ имеет единственное решение. Теорема доказана.

Число букв $a_{i}, i=\overline{1, k+1}$, участвующих в несократимой записи слова $x$, обозначим через $\omega_{x}\left(a_{i}\right)$. Пусть $\varnothing \neq A \subseteq N_{2}=\{1,2,3\}$ и

$$
H_{A}=\left\{x \in G_{2} \mid \sum_{i \in A} \omega_{x}\left(a_{i}\right) \text { четно }\right\} .
$$

Очевидно, что $H_{A}$ является подгруппой индекса 2 (см. [8]).

Пусть

$$
h_{a, j}=\left\{\begin{array}{ll}
h_{j}, & c_{a} \in H_{A}, \\
h_{j}^{\prime}, & c_{a} \in G_{2} \backslash H_{A},
\end{array} \quad j=0,1 .\right.
$$

Заметим, что

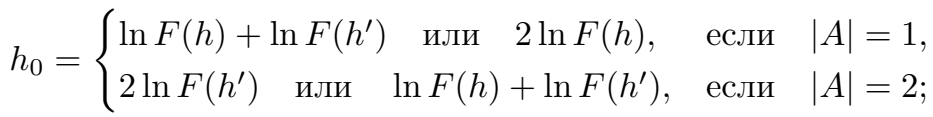

$$
\begin{aligned}
& h_{0}^{\prime}=\left\{\begin{array}{llll}
\ln F(h)+\ln F\left(h^{\prime}\right) & \text { или } 2 \ln F\left(h^{\prime}\right), & \text { если } & |A|=1, \\
2 \ln F(h) \quad \text { или } & \ln F(h)+\ln F\left(h^{\prime}\right), & \text { если } & |A|=2 ;
\end{array}\right. \\
& h_{1}=\ln F(h) \text { или } \quad \ln F\left(h^{\prime}\right) \text {; } \\
& h_{1}^{\prime}=\ln F\left(h^{\prime}\right) \quad \text { или } \quad \ln F(h) \text {. }
\end{aligned}
$$

Случай $|A|=3$ соответствует случаю $H_{A}=G_{2}^{(2)}$, изученному выше.

Теорема 5. Для модели (3) при условии (14) существуют несчетное число $H_{A}$-периодических мер Гиббса. Более того, эти меры не являются трансляиионно-инвариантными и не являются $G_{2}^{(2)}$-периодическими.

ДокАзАТЕЛьство. Система (22) имеет решение тогда и только тогда, когда $F(h)=F\left(h^{\prime}\right)$. Отсюда получаем

$$
t_{1}\left(s_{0}-t_{0}\right)+\left(t_{1}-s_{1}\right)\left(t_{0}+1\right)=0
$$

где $t_{i}=e^{h_{i}}, s_{i}=e^{h_{i}^{\prime}}, i=0,1$.

В этом уравнении обозначим $s_{0}-t_{0}=\alpha$. Тогда справедливы равенства $s_{0}=t_{0}+\alpha$, $s_{1}=t_{1}+t_{1} \alpha /\left(t_{0}+1\right)$. Из $(22)$ имеем $t_{0}=\left(1-t_{1}^{2}\right) /\left(\lambda^{2} t_{1}-1\right)$ и уравнение $\lambda^{2} t_{1}^{3}-1=0$, которое имеет единственное положительное решение $t_{1}^{*}=1 / \sqrt[3]{\lambda^{2}}$. Отсюда получаем

$$
\begin{gathered}
t_{1}=t_{1}^{*}=\frac{1}{\sqrt[3]{\lambda^{2}}}, \quad t_{0}=t_{0}^{*}=\frac{1}{\sqrt[3]{\lambda^{4}}}, \quad s_{0}=s_{0}^{*}(\alpha)=t_{0}^{*}+\alpha=\frac{1}{\sqrt[3]{\lambda^{4}}}+\alpha, \\
s_{1}=s_{1}^{*}(\alpha)=t_{1}^{*}+\frac{t_{1}^{*} \alpha}{t_{0}^{*}+1}=\frac{1}{\sqrt[3]{\lambda^{2}}}+\frac{\alpha \sqrt[3]{\lambda^{2}}}{\sqrt[3]{\lambda^{4}}+1} \quad \forall \alpha \in \mathbb{R} .
\end{gathered}
$$

Таким образом, для любого $\alpha \in \mathbb{R}$ существует решение $\left(\ln t_{0}^{*}, \ln t_{1}^{*}, \ln s_{0}^{*}(\alpha), \ln s_{1}^{*}(\alpha)\right)$ системы (22). Более того, эти решения различны при различных $\alpha$. Теорема доказана. 
Благодарности. Автор глубоко признателен профессору У. А. Розикову за постановку задачи и полезные советы по работе.

\section{Список литературы}

[1] Н. Н. Ганиходжаев, У. А. Розиков, ТМФ, 111:1 (1997), 109-117.

[2] Х.-О. Георги, Гиббсовские меры и фазовые переходы, М., Мир, 1992.

[3] К. Престон, Гиббсовские состояния на счетных множествах, Мир, М., 1977.

[4] Я. Г. Синай, Теория фазовых переходов. Строгие результаты, Наука, М., 1980.

[5] У. А. Розиков, Г. Т. Мадгозиев, ТМФ, 167:2 (2011), 311-322.

[6] П. М. Блехер, Н. Н. Ганиходжаев, ТВП, 35:2 (1990), 220-230.

[7] У. А. Розиков, ТМФ, 118:1 (1999), 95-104.

[8] U. A. Rozikov, Gibbs Measures on Cayley Trees, World Sci., Singapore, 2013.

Поступила в редакцию 29.03.2014 